\title{
Management of early pregnancy bleeding in the accident and emergency department
}

\author{
C. GILLING-SMITH, ${ }^{1}$ J. ZELIN, ${ }^{1}$ R. TOUQUET ${ }^{1} \&$ P. STEER ${ }^{2}$ \\ ${ }^{\prime}$ Department of Accident and Emergency, St Mary's Hospital, London W2 1NY, England \\ and ${ }^{2}$ Department of Obstetrics and Gynaecology, St Mary's Hospital, London W2 1NY, \\ England
}

\section{SUMMARY}

Over a period of 6 months, from February to July 1987, 26,837 patients attended St Mary's A\&E Department. Of these, $179(0.7 \%)$ were complaining of bleeding in pregnancy of less than 20 weeks gestation. In May 1987 new management guidelines were introduced. These emphasised the importance of vaginal examination and the use of ultrasound to determine the viability of the pregnancy. As a result, the incidence of admission fell from 15 of $53(28 \%)$ to 7 of $58(12 \%)$, referral to the on-call gynaecologist from 23 of $53(44 \%)$ to 13 of $58(22 \%)$ and reattendance rates from 11 of $53(15 \%)$ to 4 of $58(4 \%)$ (all changes $P<0.05)$.

\section{INTRODUCTION}

Vaginal bleeding is one of the most common complications of early pregnancy, with a reported incidence of between 16-25\% (Niswander \& Gordon, 1973; Anonymous, 1980). Accurate assessment and diagnosis at first presentation avoids (a) life-threatening complications (ruptured ectopic, intrauterine infection and septicaemia), (b) delay in definitive treatment such as the evacuation of retained products of conception (ERPC), (c) unnecessary hospitalisation, and (d) relieves patient anxiety regarding pregnancy outcome.

A recent survey on the management of threatened miscarriage in general practice suggests that current practice is often unsatisfactory, with little use being made of modern diagnostic techniques, and inappropriate management often being used (Everett et al., 1987). We felt there was a need to outline specific guidelines, particularly in assessing fetal viability, providing routine anti-D prophylaxis and advising patients.

Correspondence: $M r$ R. Touquet, Department of Accident and Emergency, St Mary's Hospital, Praed Street, London W2 1NY, England. 
There have been no studies of practice related to early pregnancy bleeding conducted in the A\&E Department despite the relatively common incidence of the problem. We therefore conducted a study of practice in the A\&E Department of St Mary's Hospital over a six month period, and assessed the effect of introducing specific guidelines for investigation and management.

The A\&E Department of St Mary's, an inner city hospital, services the eastern part of Paddington and North Kensington Health District (population 125,000) and sees 45,000 new patients a year.

\section{PATIENTS AND METHODS}

All women presenting with vaginal bleeding in pregnancy of less than 20 weeks gestation were studied. Pregnancy was confirmed by positive beta-hCG urinalysis (Rapid Absorbent Matrix Pad-RAMP or Pregnosticon test). Gestation was assessed by menstrual dates, if these were certain, or by ultrasound. Two groups of patients were studied. The management guidelines were introduced on the first day of May 1987, half way through the study period. Women seen from February to April inclusive comprise Group 1, and those seen from May to July inclusive comprise Group 2.

The following data were collected on each patient: gestation by menstrual dates, quantity and duration of menstrual bleeding, presence of abdominal pain, state of the cervical os, products of conception visible on speculum examination, presence of and adnexal mass, viability determined by ultrasound or doppler, accident doctor's dia gnosis and immediate management of the patient, whether patient was referred to on-? call gynaecologist or admitted, whether patient reattended the department within one week of initial presentation.

The patients were seen by one of nine Accident doctors, only two of whom had any postgraduate training in gynaecology. A diagnosis of inevitable or incomplete miscarriage was made if the cervical os was open or products of conception visible and the patient referred for admission and ERPC. In all other cases fetal viability was investigated by real time ultrasound-B scan (RTUS) if the gestation was between 8 and 12 weeks, or by portable doppler ultrasound with an abdominal transducer if the gestation was greater than 12 weeks. The latter was performed by the Accident doctor and followed by RTUS if no fetal heart was detected. RTUS was performed in the hospital's ultrasound department between $9.00 \mathrm{am}$ and $5.30 \mathrm{pm}$. Patients seen at night were advised to return the following morning for their scan.

RTUS will detect a gestational sac from 5 weeks to 6 weeks and a pulsating heart from 7 weeks menstrual age (Romero et al., 1984). Since our aim was to provide a rapid diagnostic assessment we limited the use of ultrasound at less than 8 weeks gestation to those patients with either a suspected ectopic pregnancy or marked vaginal bleeding and/or abdominal pain.

Patients discharged home with a diagnosis of threatened miscarriage were all serum tested and, if rhesus negative, given 250 i.u. anti-D prophylaxis intramuscularly.

The advice sheet (Figure 1) was given to the patients on discharge to correct common misunderstandings about miscarriage. We emphasised the relatively high incidence of 
WHAT IS A MISCARRIAGE?: A miscarriage is the loss of a baby during early pregnancy.

WHY DOES THE DOCTOR KEEP TALKING ABOUT ABORTIONS?: The medical term for a miscarriage is a 'spontaneous abortion'.

IS ALL BLEEDING A MISCARRIAGE?: No-most women who bleed during pregnancy are experiencing what is called a threatened abortion. Only some women with a 'threatened' go on to lose their baby.

HOW COMMON IS MISCARRIAGE?: Very common. Altogether about one fifth of pregnancies end in miscarriage, sometimes before the woman even knows she is pregnant. So you see, you are not the only one!

WHY DOES IT HAPPEN?: We do not know all the answers, but in many cases there is something very wrong with the baby so that it cannot develop properly

DOES THAT MEAN IF THE BLEEDING STOPS THERE IS AN INCREASED CHANCE MY BABY WILL BE HANDICAPPED?: No! While it is true that miscarriages often happen when the baby is abnormal, those pregnancies that continue have no more risk of producing an abnormal baby than average.

HOW DOES THE DOCTOR KNOW WHETHER IT IS A MISCARRIAGE?: The doctor will question and examine you. There are four things that are important:-

1. How much bleeding?

2. Is there any pain?

3. Has the neck of your womb opened up?

4. Is the baby all right?

If you are less than three months pregnant an ultrasound scan may be arranged to check that the baby is alive and well. If you are more than three months pregnant a special stethoscope will be used to ensure that the baby's heart is beating.

AREN'T ALL THESE 'INTERNALS' DANGEROUS?: No, although you may worry about an internal examination making the bleeding worse; if done by the doctor (or midwife) they will not affect your baby's chances. Although it can be very embarrassing to be examined, it is very important.

WILL I HAVE TO COME INTO HOSPITAL?: Not necessarily. Every woman is different, but generally if you and your baby are alright and you are not bleeding too much, there is no need for you to come into hospital.

WHY DON'T I NEED TO COME IN?: There is no special treatment for a threatened miscarriage except good old-fashioned rest. Most women prefer to rest at home with their families and friends.

WHAT DO I DO AT HOME?: Rest until at least 2 days after the bleeding has stopped. If the bleeding continues for more than a week, your GP may want to give you another check up (bleeding for a long time isn't necessarily anything to worry about).

WHAT ABOUT SEX?: You should avoid intercourse while you are resting. Some women find that sex starts the bleeding again and they have to avoid intercourse for quite a long time.

WHAT SHOULD I DO IF THE BLEEDING GETS HEAVIER?: If it gets very heavy or you pass blood clots or you get abdominal pain, you should call your GP in to check up on you and decide whether you need to come into hospital.

WHAT ABOUT FUTURE PREGNANCIES?: There is no reason why you shouldn't go on to have more children. Most women who have a miscarriage go on to have trouble free pregnancies in the future. You can get pregnant again as soon as you feel ready.

\section{REMEMBER}

Threatened miscarriages are very common. Most women go on to have healthy babies. The only treatment is rest. If you do miscarry, it doesn't affect your chances of a normal pregnancy next time.

Fig. 1 Advice sheet on miscarriage. 
early pregnancy bleeding and the reasons for it. We explained the need for an 'internal' examination and in certain cases an ultrasound or doppler. Patients were advised to rest at home for at least $\mathbf{4 8}$ hours after the bleeding had stopped, avoid intercourse and reattend the department if the bleeding continued for more than one week or became heavier.

Results were analysed by chi square test and $95 \%$ confidence limits.

\section{RESULTS}

Seventy-five patients were seen in Group 1 and 104 in Group 2. This represents $0 \cdot 6-0.7 \%$ of the department's attendance figures respectively.

Table 1 summarises the Accident doctor's initial diagnosis of patients in Groups 1 and 2. The ratio of first to second trimester bleeding was approximately 7 to 3 both at presentation and in each diagnostic category.

Table 1 Initial diagnosis of patients

\begin{tabular}{lcc}
\hline & $\begin{array}{c}\text { Group 1 } \\
\text { Feb-April 1987 }\end{array}$ & $\begin{array}{c}\text { Group 2 } \\
\text { May-July 1987 }\end{array}$ \\
\hline Total A\&E attendance & 11 915 & 14992 \\
$\begin{array}{l}\text { A\&E attendance with vaginal bleeding <20 weeks } \\
\text { pregnant }\end{array}$ & 75 & 104 \\
Viable pregnancies: & $53(71 \%)^{*}$ & $58(56 \%)$ \\
$\quad$ Total & $37(49 \%)$ & $18(17 \%)$ \\
Diagnosis on clinical exam alone & $16(21 \%)$ & $40(38 \%)$ \\
Diagnosis on clinical: exam and RTUS-doppler & $19(25 \%)$ & $46(44 \%)$ \\
Non-viable pregnancies & & $35(34 \%)$ \\
Inevitable miscarriages & & $9(9 \%)$ \\
Incomplete miscarriages & & $2(2 \%)$ \\
Complete miscarriages & $3(4 \%)$ & $0(0)$ \\
Ectopic pregnancies & $9(12 \%)$ & $46(44 \%)$ \\
Total RTUS-Scans & & \\
Booked by Accident Doctor & & \\
\hline
\end{tabular}

*\% of total in each study group

Table 2 Management and incidence of reattendance of patients

\begin{tabular}{llrr}
\hline $\begin{array}{l}\text { Threatened miscarriages } \\
\text { (diagnosed by Accident doctor) }\end{array}$ & Group 1 & Group 2 & $\chi^{2}$ \\
\hline Discharged home immediately & & & \\
Admitted for 24 h observation & $38(72 \%)^{*}$ & $51(88 \%)$ & \\
Referred to on-call gynaecologist & $15(28 \%)$ & $7(12 \%)$ & $P<0.05$ \\
Reattendance to A\&E department within 1 week & $23(44 \%)$ & $13(22 \%)$ & $P<0.05$ \\
Total & $11(15 \%)$ & $4(4 \%)$ & $P<0.05$ \\
& $53(71 \%)$ & $58(56 \%)$ & \\
\hline
\end{tabular}

*\% of total in each study group 
Table 2 records the immediate management and incidence of reattendance of the patients in both groups. Following the introduction of the guidelines, the proportion of women referred to a gynaecologist more than halved from $44 \%$ to $22 \%(95 \%$ confidence limits of fall $14 \%$ to $30 \%$ ). The proportion of women admitted for $24 \mathrm{~h}$ observation also halved from $28 \%$ to $12 \%$ ( $95 \%$ confidence limits of fall $15 \%$ to $31 \%$ ), and the proportion of women reattending the A\&E department fell by almost three quarters, from $15 \%$ to $4 \%$ (95\% confidence limits of fall $7 \%$ to $15 \%$ ).

\section{DISCUSSION}

These results show that the introduction of simple management guidelines significantly reduced admission, referral and reattendance rates in our A\&E department. A review of the literature indicates that the single most important diagnostic sign in the assessment of early pregnancy bleeding is the presence of a pulsating fetal heart (Hertz, 1984). This may be detected by RTUS from the 7th week of gestation and by doppler ultrasound from the 12th week of gestation (Robinson, 1972). In its presence the overall successful pregnancy outcome is reported as $96 \%$ with a range from $80-100 \%$ depending on the presence or absence of subchorionic bleeding respectively. In the presence of an adnexal mass and no demonstrable intrauterine pregnancy on ultrasound the diagnosis of ectopic pregnancy can be made with $95 \%$ certainty (Robinson \& DeCrespigny, 1983).

Our results in Table 1 are consistent with previous studies in showing that the majority of patients with threatened abortion present between 7 and 10 weeks gestation and that approximately half the patients with first trimester bleeding continue with a normal pregnancy (Stabile et al., 1987).

Routine sonographic examination at the initial presentation to the Accident department has several distinct advantages. Patients with a proven viable pregnancy can be discharged with a confident prognosis and are thus less likely to return to the department at the slightest hint of abdominal pain or vaginal spotting.

They are not kept waiting in the department to be seen by the on-call gynaecologist (whose first priority is often a busy labour ward) prior to discharge home. Admission to hospital for bed rest, of no proven value in threatened abortion, (Diddle et al., 1953) is avoided. Cases of missed abortion are diagnosed early, and given definitive treatment, reducing the incidence of haemorrhage and sepsis.

Prior to the introduction of the guidelines, bimanual pelvic examination was not performed routinely on all patients by the Accident doctor (11\% of cases in Group 1). A similar problem is encountered in patients referred by general practitioners. The misconception that internal examinations may aggravate the bleeding or cause the patient to abort is unfounded and we clarified this point in our advice sheet. On the contrary, early assessment of the state of the cervix and removal of products of conception from the os reduces the incidence of haemorrhage and shock. Finally pelvic examination is mandatory in the diagnosis of an adnexal mass in suspected ectopic pregnancy since only $4 \%$ of these can be demonstrated on ultrasound.

The RAMP assay is a sensitive monoclonal antibody-based enzyme immunoassay for the qualitative detection of human chorionic gonadotrophin (beta hCG) in urine. The assay will detect levels of beta hCG from 10 days post-conception. It is a simple five 
minute test and is particularly helpful in the A\&E department in cases of suspectedô ectopic pregnancy in which the routine pregnosticon test has proved negative $\underset{\mathrm{D}}{\mathrm{B}}$ (McCready et al., 1978).

Before introduction of the guidelines, anti-D prophylaxis was not routinely given to $\vec{s}$ patients discharged home. The risk of maternal immunisation following miscarriageo ranges from $2-9 \%$ depending on gestational age, 8 weeks to 12 weeks respectively (Freda et al., 1970; Litwak et al., 1970). Since the risk of sensitisation through $\frac{\vec{p}}{\bar{\sigma}}$ transplacental bleeding is almost twice as high in threatened abortion, we included in $\frac{0}{2}$ our guidelines the recommendation that prophylactic anti-D $250 \mathrm{i}$.u. be given intramuscularly to all rhesus negative patients with threatened abortion within 72 hours of the $\vec{\circ}$ first bleed.

It is important to remember that for a large proportion of Accident doctors, this job is $\frac{\rho}{\rho}$ part of their postgraduate training in surgery, medicine or general practice. Subse- 3 . quently they may not get any further training in gynaecology and we feel our guidelines will be of value in their subsequent specialities, in particular general practice.

We have used the term miscarriage rather than abortion throughout, as in our advice $\stackrel{\vec{\omega}}{\omega}$ sheet. Many patients are particularly sensitive to this, regarding abortion as meaning 9 termination.

In summary the introduction of simple management guidelines for patients with vaginal bleeding was shown to improve both clinical practice and communication with the patient by the use of an advice sheet.

\section{REFERENCES}

Anon. (1980) Vaginal bleeding in early pregnancy (editorial). British Medical fournal 281, 470.

Diddle A. W., O’Connor K. A. \& Jack R. (1953) Evaluation of bed rest in threatened abortion. Obstetrics and Gynaecology 21, 63-67.

Everett C., Ashurst H. \& Chalmers I. (1987) Reported management of threatened miscarriage by general $\stackrel{\overline{\mathrm{O}}}{\exists}$ practitioners in Wessex. British Medical fournal 295, 583-6.

Freda V. J., Gorman J. G., Galen R. S. \& Treacy N. (1970) The threat of Rh immunisation from abortion Lancet 2, 147.

Hertz J. B. (1984) Diagnostic procedures in threatened abortion. Obstetrics and Gynaecology 64, 223-9.

Litwak O., Taswell H., Banner E. \& Keith L. (1970) Fetal erythrocytes in maternal circulation after spontaneous abortion. Fournal of the American Medical Association 214, 531.

McCready J., Braunstein G. D., Helm D. \& Wade M. E. (1978) Clinical Chemistry 24, 1958-61.

Niswander K. \& Gordon M. (1973) The Collaborative Perinatal Study: the women and their pregnancies. W. B. Saunders, Philadelphia.

Robinson H. P. \& De Crespigny L. (1983) Ectopic pregnancy. Clinics in Obstetrics and Gynaecology 10, 407-윽

421.
Robinson H.P. (1972) Detection of fetal heart movement in first trimester of pregnancy using pulsed ultrasound. British Medical fournal 4, 466-468.

Romero R., Jeanty P. \& Hobbins J. C. (1984) Diagnostic ultrasound in the first trimester of pregnancy. Clinics in Obstetrics and Gynaecology 27, 286-313.

Stabile I., Campbell S. \& Grudzinskas J. G. (1987) Ultrasonic assessment of complications during first trimester of pregnancy. Lancet ii, 1237-40. 